\title{
Use of medicines in palliative care
}

\section{Franco De Conno}

For centuries the essence of palliative care was a central point of medicine, even if the term was not yet used. Having few means to cure the patient, the 'treatment' concentrated more on the person, trying to give comfort, support and attention to dignity. With the introduction of so called modern medicine, there was a shift in attention from the whole patient to the disease or pathology only. In the technology oriented society of the years 1950-1970, dignity and quality of life were not high on the agenda. Communication between healthcare professionals and patients and their families was limited and poor.

In this context, palliative care was born with the creation of the St Christopher's Hospice in the UK. The ambition of its founder, Cicely Saunders, was to integrate 'a scientific programme concerned with the discriminating use of drugs with the tender loving care provided within those other institutions. ${ }^{1}$ Her definition of palliative care did not mean to renounce science and technology, but combine 'high tech with

\section{Correspondence to Dr F De Conno,} Honorary director of the EAPC, Honorary chairman of the ResNet of the EAPC, Via Venezian 1, Milano, Italy, 20133; Franco.DeConno@istitutotumori.mi.it high touch' attention to the suffering of the human being. The centrality of the patient and his/her family was a core argument which also included the collaboration of various healthcare professionals to deliver best care for the patient. Pharmacists were an important part of such a multiprofessional team.

The first drugs used for the relief of pain were galenic preparations, and the remedies used for symptom control were often off label medications. The collaboration with some hospital pharmacists was fundamental for the development of certain treatments in palliative care. Today we have a wide range of pharmacological preparations at our disposal to control the many debilitating symptoms of our patients with advanced disease but the list of drugs is still not complete and we may need to use off label drugs to control certain conditions. Good collaboration between physicians and pharmacists remains of great importance to be able to offer the best relief to our patients, and I am very happy to introduce a series of interesting papers giving more insight into different subjects on the care of patients with incurable disease.

The list of essential medicines in palliative care represents a fundamental instrument for governments to offer their population the drugs that are absolutely necessary to obtain good care. The importance of this list is underlined in the paper by Nathan Cherny 'How well are we doing in treating cancer pain in Europe' that shows that there are still many countries were the most important drugs are not available or only available with great difficulty.

In another article, a very specific item of palliative medicine is dealt with-the care of the dying and the last days of life. The experience of the traumatic departure of one's loved ones can influence the rest of the lives of family members.

Detailed knowledge about symptoms and the instruments to control them and careful assessment of the patient form the basis for successful care. Two of the series of papers deal with depression and breakthrough pain. Often underestimated or even unrecognised, these symptoms can harm the quality of life of a patient considerably.

I very much appreciate the initiative of the editor to offer this insight into palliative care and wish you good reading.

\section{Competing interests None.}

Provenance and peer review Commissioned; not externally peer reviewed.

\section{Reference}

1. Saunders C. Hospice care. Am J Med 1978;65:726-8. 